\title{
Pyridoxine-induced toxicity in rats: a stereological quantification of the sensory neuropathy
}

\author{
Tracy Ann Perry ${ }^{\mathrm{a}, *}$, Ananda Weerasuriya ${ }^{\mathrm{b}}$, Peter R. Mouton ${ }^{\mathrm{c}}$, \\ Harold W. Holloway ${ }^{a}$, Nigel H. Greig ${ }^{a}$ \\ ${ }^{a}$ Section of Drug Design and Development, Laboratory of Neurosciences, Gerontology Research Center, \\ National Institute on Aging, National Institutes of Health, Baltimore, MD 21224, USA \\ ${ }^{\mathrm{b}}$ Division of Basic Medical Science, Mercer University School of Medicine, Macon, GA 31207, USA \\ ${ }^{\mathrm{c}}$ Laboratory of Experimental Gerontology, Gerontology Research Center, National Institute on Aging, National Institutes of Health, Baltimore, \\ MD 21224, USA
}

Received 25 March 2004; revised 16 June 2004; accepted 21 July 2004

\begin{abstract}
Excess ingestion of pyridoxine (vitamin B6) causes a severe sensory neuropathy in humans. The mechanism of action has not been fully elucidated, and studies of pyridoxine neuropathy in experimental animals have yielded disparate results. Pyridoxine intoxication appears to produce a neuropathy characterized by necrosis of dorsal root ganglion (DRG) sensory neurons and degeneration of peripheral and central sensory projections, with large diameter neurons being particularly affected. The major determinants affecting the severity of the pyridoxine neuropathy appear to be duration and dose of pyridoxine administration, differential neuronal vulnerability, and species susceptibility. The present study used design-based stereological techniques in conjunction with electrophysiological measures to quantify the morphological and physiological changes that occur in the DRG and the distal myelinated axons of the sciatic nerve following pyridoxine intoxication. This combined stereological and electrophysiological method demonstrates a general approach that could be used for assessing the correlation between pathophysiological and functional parameters in animal models of toxic neuropathy.
\end{abstract}

Published by Elsevier Inc.

Keywords: Sensory neuropathy; DRG; Sciatic nerve; H reflex; M wave; Axonopathy

\section{Introduction}

Vitamin $\mathrm{B}_{6}$ is a dietary requirement of nonruminant animals and is a coenzyme in many important biological reactions. The vitamin has three natural occurring forms: pyridoxol (pyridoxine), pyridoxal, and pyridoxamine. It is a relatively simple compound with three substituted pyridine derivatives that differ only in the functional group in the four position: alcohol (pyridoxine or pyridoxol), aldehyde

* Corresponding author. Section of Drug Design and Development, Laboratory of Neurosciences, Gerontology Research Center, National Institute on Aging, National Institutes of Health, 5600 Nathan Shock Drive, Baltimore, MD 21224. Fax: +1 4105588323.

E-mail addresses: tracyann.perry@amylin.com (T.A. Perry), greign@nia.nih.gov (N.H. Greig) (pyridoxal), or amine (pyridoxamine). Of these, pyridoxine is most commonly used as a dietary supplement and therapeutic agent. High doses of pyridoxine have been used to treat conditions such as premenstrual or carpal tunnel syndromes, and as therapy for intoxication secondary to the false morel mushroom Gyromitra esculenta (Hanrahan and Gordon, 1984). The rationale supporting this is that the active toxin monomethylhydrazine competitively inhibits a pyridoxine-dependent step in the synthesis of the neurotransmitter gamma-aminobutyric acid.

While pyridoxine deficiency is manifested by distal, predominantly sensory neuropathy, pyridoxine has also been identified as a neurotoxicant. During the 1980s, attention was drawn to a neurologic disease, which presented in individuals consuming large quantities of 
vitamin $\mathrm{B}_{6}$ for prolonged periods of time. Schaumburg et al. (1983) has described a severe sensory neuropathy of insidious onset and course associated with chronic abuse of oral pyridoxine supplements. The recommended oral daily dose is $2-4 \mathrm{mg}$ in human adults. Daily oral doses of up to $6 \mathrm{~g}$ for $12-40$ months lead to a progressive sensory neuropathy manifested by sensory ataxia, diminished distal limb proprioception, paresthesia, and hyperesthesia (Dalton and Dalton, 1987; Foca, 1985; Schaumburg and Spencer, 1979; Schaumburg et al., 1983). Parry and Bredesen (1985) subsequently reported that as little as $200 \mathrm{mg}$ of pyridoxine per day could induce this syndrome. It has been successfully reproduced in both acutely and chronically treated dogs (oral dosages of $50-300 \mathrm{mg} \mathrm{kg}^{-1}$ day $^{-1}$ for up to 112 days) (Hoover et al., 1981; Krinke et al., 1980; Montpetit et al., 1988; Schaeppi and Krinke, 1982) as well as rats (Bowe and Veale, 1988; Krinke and Fitzgerald, 1988; Krinke et al., 1985; Nisar et al., 1990; Windebank et al., 1985; Xu et al., 1989), and appears to be secondary to a reversible sensory nerve axonopathy at low and intermediate doses and an irreversible sensory ganglion neuropathy at high doses (Krinke et al., 1980, 1985; Phillips et al., 1978; Schaeppi and Krinke, 1982; Windebank et al., 1985). In rat studies, three intraperitoneal dosing regimens were generally employed, as follows: short term/high dose $1200 \mathrm{mg} \mathrm{kg}-1$ day $^{-1}$ for 1-15 days (Krinke and Fitzgerald, 1988; Xu et al., 1989); intermediate dosing, $600 \mathrm{mg} \mathrm{kg}^{-1}$ day for $1-15$ days (Krinke et al., 1985; Xu et al., 1989); long term/low dose, $100-300 \mathrm{mg} \mathrm{kg}^{-1} \mathrm{day}^{-1}$ for up to 12 weeks (Krinke and Fitzgerald, 1988; Windebank et al., 1985; Xu et al., 1989). Such experimental studies confirmed the morphologic pattern of peripheral nervous system lesions of pyridoxine neurotoxicity, reflecting primary injury to the cytons of neurons residing in peripheral sensory (dorsal root, trigeminal) ganglia, with secondary degeneration of axons of affected cells. These are some of the largest neurons with long processes, and hence represent cells with the greatest metabolic susceptibility. The cell body changes are manifested in cytoplasmic alterations, such as vacuolization, neurofilament aggregates, and chromatolysis. More advanced neurotoxic changes lead to neuronal death with accompanying phagocytosis by satellite cells.

Compelling evidence exists in dogs (Schaeppi and Krinke, 1982), rodents (Xu et al., 1989), and in humans (Albin et al., 1987) relating to the functional and physiological parameters induced by pyridoxine neurotoxicity. However, the anatomical and morphological anomalies within the spinal cord and peripheral nerves resulting from excessive doses of pyridoxine have to date been solely subjective. Quantitative studies, involving information about number and size of axons within a particular nerve, or the size and distribution of cell bodies within specific dorsal root ganglia (DRG), are invaluable for studying developmental, experimental, and pathological changes. Design-based stereological techniques (Mouton, 2002) allow for precise and unbiased estimates of both number and sizes of axons from a small population. Indeed, a rapidly emerging literature exists that uses these techniques in peripheral nerves and nerve roots (Larsen, 1998; Larsen et al., 1998; Schionning and Larsen, 1997; Schionning et al., 1998a,b). Using design-based stereological techniques, this study has quantified the morphological changes that occur in the dorsal root ganglia and the sciatic nerve as a result of pyridoxine toxicity. This approach involving morphology correlates of sensory and motor nerve conduction velocity provides a general methodology for assessment of animal models of toxic neuropathy.

\section{Materials and methods}

The study was undertaken using 14 adult male Sprague-Dawley rats weighing approximately $300-350 \mathrm{~g}$ each. Animals were housed under controlled light-dark and temperature conditions with food and water available ad libitum. The rats were randomly assigned to two groups with half of the rats receiving pyridoxine injections and the rest receiving vehicle injections. Pyridoxine solution was prepared immediately before each injection. Pyridoxine (Sigma) was diluted in sterile distilled water, $\mathrm{pH}$ corrected to 7.2 , warmed, and administered at $400 \mathrm{mg} \mathrm{kg} \mathrm{kg}^{-1}$ intraperitoneally (ip) twice a day, morning and evening, for 14 days. The dose of pyridoxine used in this study was based on prior unpublished evaluation of pyridoxine toxicity.

\section{Electrophysiology}

Nerve conduction studies in the sciatic nerve were performed 13-14 days after the start of pyridoxine treatment. Rats were anaesthetized with ketamine $(90 \mathrm{mg}$ $\left.\mathrm{kg}^{-1}\right)$ and acepromazine $\left(0.91 \mathrm{mg} \mathrm{kg}{ }^{-1}\right)$. Rectal temperature was maintained at $37^{\circ} \mathrm{C}$ and intramuscular temperature at between $33^{\circ} \mathrm{C}$ and $34^{\circ} \mathrm{C}$. A recording electrode was inserted between the 4th and 5th digits, parallel to the long axis of the foot. A ground electrode was inserted into the muscle of the thigh. External stimulation at the ankle and at the sciatic notch was done using a bipolar electrode, followed by near nerve stimulation at the ankle and at the sciatic notch through a monopolar cathodal electrode. An equivalent anode electrode was inserted into the calf muscle $1 \mathrm{~cm}$ proximal to the cathode. Data were acquired and analyzed using a PowerLab system (ADInstruments, Australia).

The position of the active recording electrode was adjusted to maximize the $\mathrm{M}$ wave amplitude. For each external stimulation, adjustments in the position of the stimulating electrode were made to bring thresholds for evoked $\mathrm{H}$ or $\mathrm{M}$ waves at or below 15 and $5 \mathrm{~mA}$, respectively. For each near nerve stimulation, the position of the stimulating electrode (at the notch and the ankle) was adjusted to bring thresholds to evoke $\mathrm{H}$ or $\mathrm{M}$ waves at or 
below $1.5 \mathrm{~mA}$. Inflexion-to-inflexion and peak-to-peak amplitudes of $\mathrm{H}$ and $\mathrm{M}$ waves were measured. Sensory and motor conduction velocities were calculated as the difference in response latencies following stimulation at the sciatic notch and the ankle, divided by the distance between the two locations. Latencies were measured to the first inflexion and major peak for the $\mathrm{M}$ or $\mathrm{H}$ waves. Two separate measurements of $\mathrm{M}$ and $\mathrm{H}$ wave amplitudes in response to stimulation at the ankle and the sciatic notch were made, one external and one near nerve.

\section{Histopathology and design-based stereological evaluation}

On day 14, all animals were sacrificed and segments of sciatic nerve from just below the notch and lumbar dorsal root ganglia (L4-L6) were taken for histological analysis and stereological quantification. Tissues were post-fixed overnight in $4 \%$ paraformaldehyde before embedding in paraffin and serial sectioning at 4-6 $\mu \mathrm{m}$ in the transverse (coronal) plane.

Representative sciatic nerve and dorsal root ganglion sections were deparaffinized and stained with luxol fast blue for myelin combined with a light cresyl violet counterstain. After deparaffinization, sections were boiled in citrate buffer, $\mathrm{pH} 6.0$ for 20-25 min. After cooling, sections were washed in tap water, followed by several washes in phosphate-buffered saline and blocked in goat serum overnight at $4^{\circ} \mathrm{C}$. Adjacent sections were stained immunocytochemically using the polyclonal rabbit anti-neurofilament antibodies (Chemicon, International Inc., Temecula, CA) against the $68-\mathrm{kDa}$ neurofilament fragment (NF-L), the 145$\mathrm{kDa}$ C-terminal neurofilament chain (NF-M), and the extreme neurofilament C-terminus (NF-H) at 1:500 dilution. Visualization of positive immunoreactivity was carried out using avidin-biotin/horse radish peroxidase and DAB as the chromogen. Stained sections were dehydrated in an ascending series of alcohols before mounting in D.P.X. (VWR Scientific Products, Willard, $\mathrm{OH}$ ).

\section{Sciatic nerve stereology}

Number and area estimation of the nerve fibers using neurofilament-positive immunoreactivity as the axonal marker

Morphometric analyses were performed with the aid of a computerized stereology system powered by Stereologer software (Systems Planning and Analysis Inc., Alexandria, VA) and hardware consisting of a Zeiss Axioskop microscope, motorized XYZ stage (Applied Scientific Instrumentation, Eugene, OR), Sony CCD video camera, TARGA video card, and personal computer/monitor. The user outlined the reference space (defined as the cross section of myelinated nerve) and the system moved the stage in a systematic-random manner across the delineated area. Sampling frames of area $\mathrm{a}$ (frame) $\left[\mathrm{a}(\right.$ frame $\left.)=50 \mu \mathrm{m}^{2}\right]$ were moved in steps of area $\left(x\right.$-step $\cdot y$-step $\left.=100 \mu \mathrm{m}^{2}\right)$ across the cross-sectional area of the nerve in a raster pattern. Thus, the area sampling fraction on each cross section was $50 / 10,000=1 / 200$.

The parameters of interest were the area of myelinated axons stained with luxol fast blue (myelin + axon) and neurofilament-positive axons. The axonal area fraction $A_{\text {axon }} / A_{\text {sampled }}$ and total area of axons $A_{\text {total }}$ for crosssections of sciatic nerve biopsies were estimated using point counting stereology, as shown in Fig. 1, using methods detailed previously (Larsen, 1998; Mouton, 2002). For area fraction, areas were calculated as the ratio of the products of the sum of points that hit the structure $\Sigma P$ and the area associated with each point in the grid, $a(p)$ :

Area fraction $=\frac{A_{\text {axon }}}{A_{\text {sampled }}}=\frac{\Sigma P_{\text {axon }} \bullet a(p)}{\Sigma P_{\text {sampled }} \cdot a(p)}$

The total axonal area $A_{\text {total }}$ was calculated as the product of the area fraction $A_{\text {axon }} / A_{\text {sampled }}$ at high magnification $(40 \times)$ and the total nerve area Total $A_{\text {nerve }}$ at low magnification $(5 \times)$, where

Total $A_{\text {nerve }}=\Sigma P_{\text {nerve }} \bullet a(p)$

Because equivalent shrinkage occurred in both the denominator of $A_{\text {axon }} / A_{\text {sampled }}$ and Total $A_{\text {nerve }}$, their product shrinkage cancels out, leading to an unbiased estimate of total axon area $A_{\text {total }}$ :

$A_{\text {total }}=A_{\text {axon }} / A_{\text {nerve }} \cdot$ Total $A_{\text {nerve }}$

\section{Local size estimation of individual axons}

The local size estimators presented below are two-step procedures with a sampling step followed by an estimation step. In the first step, a systematically uniform random sample of axon profiles is obtained, and in the second step the local size estimation is carried out on the sampled axon profiles. Estimation of the mean cross-sectional area of the myelinated fibers was based on the 2-1 rotator principle (Jensen and Pakkenberg, 1993; Jensen Vedel and Gundersen, 1993; Mouton, 2002). A central location within each axon was marked and a line random in $360^{\circ}$ was generated across the diameter of the axon. Three test lines were placed at systematic-random locations perpendicular to the random diameter. The points of intersection between the axon boundary and the test lines were marked, and the software calculated an estimate of the profile area as $\pi l^{2}$, where $l$ is the length of each test line from the random diameter to the profile boundary. The estimator is unbiased, irrespective of the position of the fixed point or the shape of the axon (Jensen Vedel and Gundersen, 1993).

\section{Total myelin area}

The total area of myelin, including any space between the myelin sheath and the axon, was calculated by subtraction 


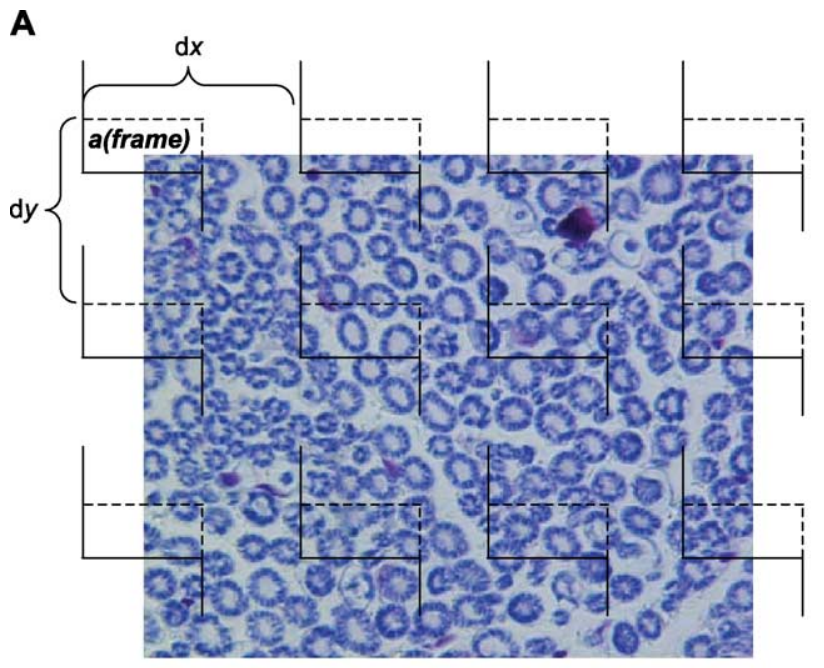

B

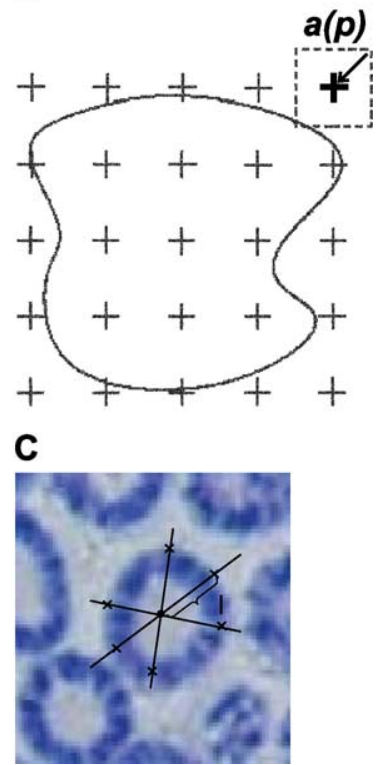

Fig. 1. The fractionator principle, point counting technique, and procedure for estimating individual myelinated axon area using the two-dimensional nucleator. In the fractionator principle (A), the axon profiles are counted in an unbiased counting frame of area a(frame) for each sampling area, $\mathrm{a}($ frame $)=\mathrm{d} x \cdot \mathrm{d} y$. The solid lines of the counting frame are the inclusion lines and the dashed lines are the exclusion lines. Only profiles within the counting frame or touching the inclusion lines are counted. The first counting frame is placed randomly, and the succeeding frames are systematically displaced by $\mathrm{d} x$ or $\mathrm{d} y$. Estimates of the total number of myelinated axons are calculated as the product of the number of axons counted in the known fraction and the inverse sampling fraction, that is, $a$ (sample) / $a$ (frame). The profile area can be estimated by placing a grid of systematically tested points randomly on the profile of interest. The number of points that hit the profile area (consistently confining the observations to one corner of the cross multiplied by the area associated with each grid point a(p)) is an unbiased estimate of the area. The two-dimensional nucleator method is used for estimating the area of individual myelinated axons (C). The approximate center of the axon is marked and the computer generates several right-angled lines from that point. The points of intersection with the boundary and the test lines are marked, and the computer calculates the estimate of the profile area as $\pi l^{2}$, where $l$ is the length of each test line from the center to the profile boundary.

of the neurofilament area (estimated on neurofilamentstained sections) from the total area of myelinated axons (estimated on luxol fast blue sections).

Approximately 200 locations in the $x-y$ plane of each nerve were sampled. The observed relative interanimal variance of the area estimates, that is, the coefficient of variation $\mathrm{CV}(\mathrm{obs})$ was calculated as the standard deviation of the estimates of the number SD divided by the group mean $\left(\sum / n\right), \mathrm{CV}(\mathrm{obs})=\mathrm{SD} /\left(\sum / n\right)$. Mean estimator precision, the mean coefficient of error for each group was $<0.10$ (mean $\mathrm{CE}<10 \%$ ) based on independent sampling of axons within each nerve.

\section{Dorsal root ganglion stereology}

Dorsal root ganglion sections were stained with luxol fast blue and cresyl violet. Classifications for neurons in the DRG were based on their size, appearance, and histochemical reactions, leading to the designation $\mathrm{A}$ and $\mathrm{B}$ cells, according to the following description:

A-cells Profiles of these cells have cytoplasm with welldefined Nissl granules on a light background. Large islands of less intensely stained material are common, with a more coarsely granular or clumped appearance, while some have smaller more numerous islands. The nucleus is generally lightly stained, usually with one large, intensely stained central nucleolus.

B-cells Profiles of this cell type have a dark cytoplasm that is more homogeneous and intensely stained than A-cells. The homogeneity of the cytoplasmic staining can be variable, with some cells exhibiting uniform staining throughout the cytoplasm, while others show a more dense distribution toward the outer perikaryon and a lighter more diffuse central staining. In all the B-cells, the nucleolus is light and very often has multiple nucleoli located peripherally in addition to many smaller chromatin condensations.

\section{Total area of A- and B-cells}

The combined area of the A- and B-cells was quantified in two sections and the average value reported. No distinction was made between the A- and B-cells. As detailed above, the total mean area of DRG cells was estimated as the product of the area fraction for A- and Bcells at high magnification $(63 \times)$ and the total DRG area estimated at low magnification $(5 \times)$.

\section{Statistical analysis}

Stereological parameters were subjected to a one-way analysis of variance (ANOVA). Animal weights were 
compared across time using repeated measures ANOVA. For the electrophysiology, data independent sample $t$ tests for equality of means assuming equal variances were carried out on the sensory and motor nerve conduction velocities where appropriate. The level of significance was set at $P<$ 0.05 . The following symbols were used in the figures to express the level of significance. ${ }^{*} P<0.05$; $* * P<0.01$; and $* * * P<0.001$

\section{Results}

\section{Effect of pyridoxine intoxication}

Animals were injected twice daily with either $400 \mathrm{mg}$ $\mathrm{kg}^{-1}$ pyridoxine or vehicle for 2 weeks. All animals in the pyridoxine-treated group showed immediate signs of discomfort, as evidenced by increased unsteadiness, vocalizing, and aggressiveness toward its cage-mate. Vehicleinjected rats exhibited no effects of treatment. The severity of the unsteadiness in the pyridoxine-treated group progressed during the injection period into full body writhing and full extension of the hind limbs with temporary muscle rigor. Although these effects were temporary, lasting between 10 and $20 \mathrm{~s}$, the severity increased with accumulating doses of pyridoxine.

Aside from the temporary injection-related effects in the pyridoxine-treated group, no overt signs of more permanent functional damage appeared during the first week; both injection groups appeared indistinguishable. However, during the second week, functional deficits in the pyridoxine-intoxicated rats became overtly apparent. The general unsteadiness, initially only evident postinjection, progressed to a permanent, pronounced hindlimb deficit. The deficit progressed within 7 days to include the forelimbs, leaving the animal unable to coordinate all four limbs simultaneously, resulting in a severe motor ataxia. Although the ataxia was severe, the animals were able to move around the cage by adopting a shuffling gait, likely as a result of the preserved muscle force. The majority of the pyridoxine-treated animals maintained an intact righting reflex. When placed on their backs, these rats could correct their position by vigorous tail swinging combined with paddling movements of the hind limbs (see Fig. 2 for sequential series of photo images). Pyridoxine-treated animals exhibited a curling of the toes on the hind paws, which contributed to the ataxia. Although care was taken to ensure the animals could reach both food and water, as shown in Fig. 3, pyridoxine intoxication caused a $20 \%$ reduction in body weight following the second week of injections.

\section{Electrophysiology}

Five animals were studied in the pyridoxine-treated group during maximum neurological abnormality on days
12-14 and four rats in the vehicle-injected group at the same time point.

\section{Sensory nerve conduction}

Pyridoxine treatment caused a marked attenuation of the $\mathrm{H}$ reflex. Fig. 4 shows an example of an EMG potential recorded from one vehicle-injected animal and one pyridoxine-injected animal. Of the five animals in the pyridoxine group, three had no consistently detectable $\mathrm{H}$ waves and the remaining two animals showed substantial reductions in $\mathrm{H}$ wave amplitude. Values for sensory nerve conduction velocity [mean $\pm \mathrm{SD}$ (in $\mathrm{m} \mathrm{s}^{-1}$ )] measured from peak-topeak were as follows: vehicle, $45.38 \pm 10.69(n=4)$; pyridoxine, $30.10 \pm 2.69(n=2)$; for near nerve stimulation and vehicle, $41.25 \pm 9.55(n=4)$; pyridoxine, $22.10 \pm 8.63$ $(n=2)$ for surface stimulation. Overall sensory conduction velocity was lower in the pyridoxine-injected group than in the vehicle-injected control group; however, due to the low number of pyridoxine animals with observable $\mathrm{H}$ waves, conduction velocity could not be calculated, and therefore no statistical analyses were carried out.

\section{Motor nerve function}

Neither the amplitude of the motor EMG response (M wave) nor the motor conduction velocity varied between vehicle and pyridoxine groups. Values for motor nerve conduction velocity (mean $\pm \mathrm{SD}$ (in $\left.\mathrm{m} \mathrm{s}^{-1}\right)$ ) measured from peak-to-peak were as follows: vehicle, $39.12 \pm 9.66(n=4)$; pyridoxine, $34.06 \pm 3.47(n=5)$; for near nerve stimulation $(t=1.103, P>0.05)$; and vehicle, $39.40 \pm 5.50(n=4)$; pyridoxine, $31.82 \pm 4.27(n=5)$ for surface stimulation $(t=$ 2.335, $P>0.05)$. The results of EMG recordings during peripheral nerve stimulation in pyridoxine-intoxicated rats were consistent with selective toxicity to sensory but not motor nerve function.

\section{Morphological evaluation of pyridoxine intoxication}

After 14 days of pyridoxine treatment, the integrity of individual myelinated fibers and axons within the sciatic nerve was visualized using luxol fast blue histochemistry and neurofilament immunocytochemistry. Fig. 5 illustrates the microscopic appearance of typical sciatic nerve sections from a pyridoxine-treated animal (Figs. 5A-B) and a vehicletreated animal (Figs. 5D-E). There were marked differences in the appearance of the myelinated fibers stained with luxol fast blue in pyridoxine-treated rats (Fig. 5A) compared to that in vehicle-treated rats (Fig. 5D). Sections from pyridoxinetreated rats showed fewer large diameter fibers and a relative increase in the number of smaller diameter fibers, resulting in an apparent decrease in nerve fiber density and an increased endoneurial area (the interstitial connective tissue surrounding the fibers) as compared to sections through sciatic nerves of vehicle-treated rats. 

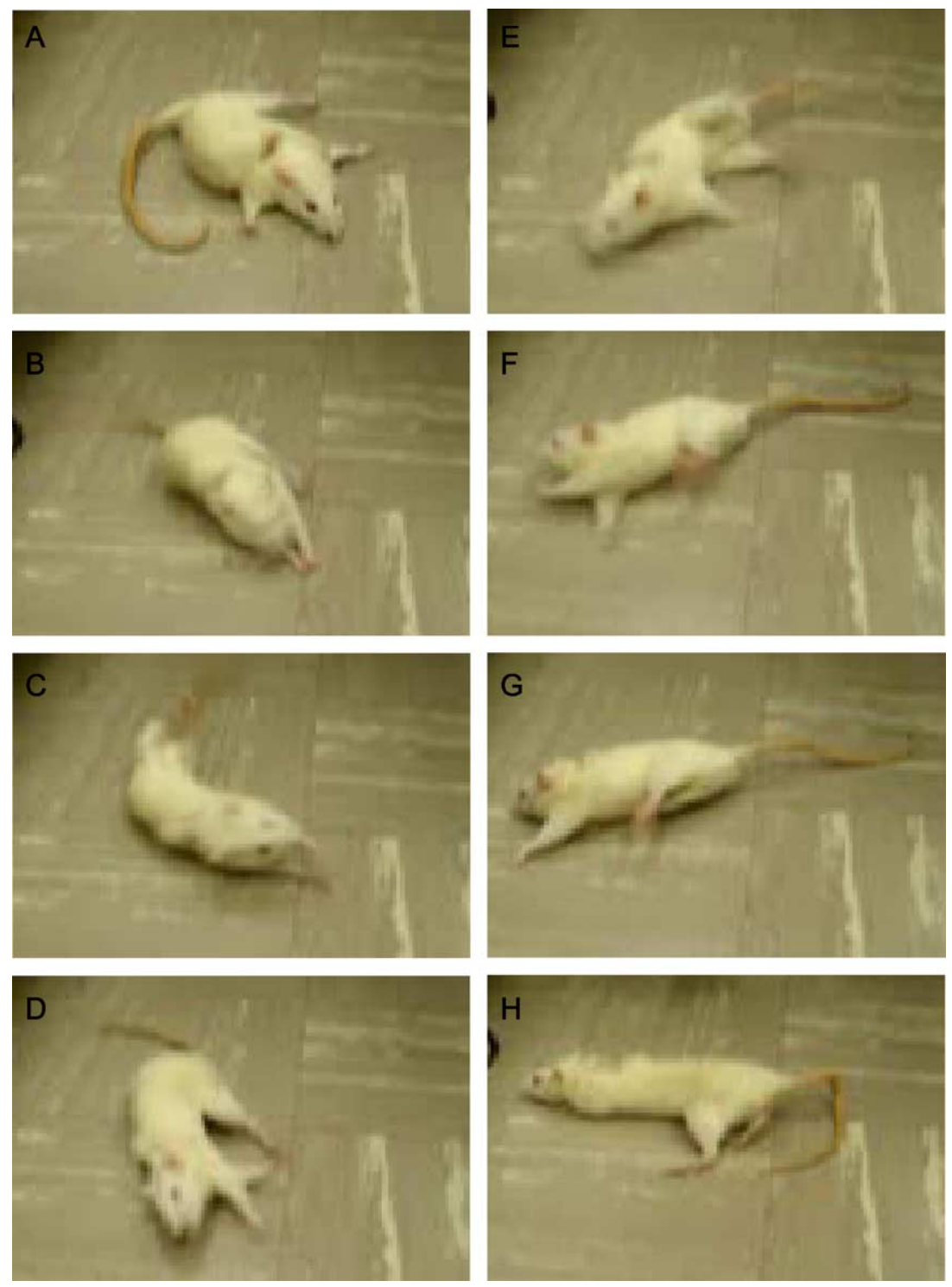

Fig. 2. Sequential photo images of the pyridoxine induced ataxia after 14 days of treatment. A rat placed directly on a flat surface (A) changes its direction of movement by a vigorous swinging of the tail (B-C), paddling movements of the front $(C-G)$ and hind $(\mathrm{D}-\mathrm{G})$ legs, and a full body twist / roll. The rat is unable to walk or stand normally on all four paws $(\mathrm{H})$. When motionless, the rat rests on its abdomen with the front limbs splayed apart (A) and implements a range of flailing motions to coordinate movement.

Fig. 5 shows neurofilament-positive immunoreactivity, a specific marker for axons, in typical sciatic nerve crosssections from pyridoxine- and vehicle-treated animals. Axonal degeneration is evidence by an increase in the number of irregularly shaped profiles in pyridoxineintoxicated animals (panel B). Consistent with the luxol fast blue-stained sections, the endoneurial area appeared larger in pyridoxine animals, likely as a result of the increase in smaller diameter, irregular-shaped neurofilament-positive axons.

Similar evidence of cellular degeneration was evident in the DRG of pyridoxine-treated rats. The pyridoxine animals showed an abundance of irregular-shaped A- and B-cells (Fig. 5C), and a more diverse size distribution than the vehicle-injected animals (Fig. 5F). The degener- ating neurons displayed morphological features characteristic of apoptosis, which was absent in vehicle-injected rats, and fewer A- and B-cells in the pyridoxine animals. The luxol fast blue staining for myelinated fibers and neurofilament immunoreactivity for axonal staining in the sciatic nerve sections, and the cresyl violet-stained A- and B-cells in the DRG sections, were quantified using design-based stereology.

Stereological quantification of sciatic nerve morphology following pyridoxine intoxication

Table 1 illustrates the stereological parameters examined in vehicle- and pyridoxine-treated rats. The pyridoxine-treated group showed significant increases 


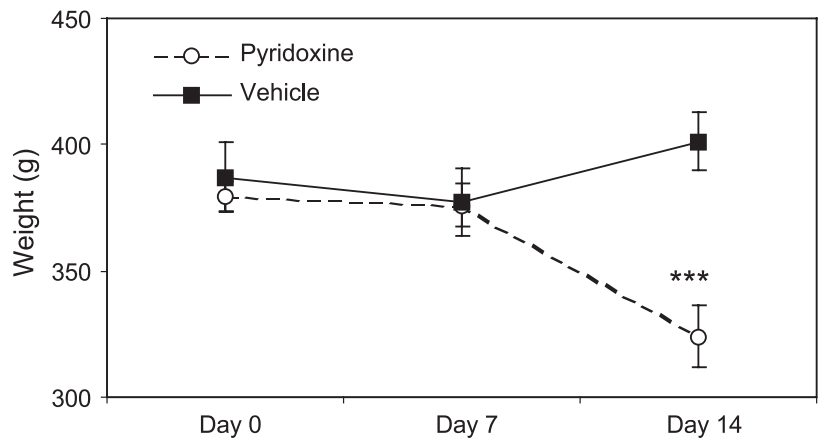

Fig. 3. Weight in grams of pyridoxine- and vehicle-injected animals over the 2-week injection schedule. Animal weights were collated and are presented at day 0 (weight before the onset of the injections), day 7 , and day 14 (day of sacrifice). Pyridoxine-intoxicated rats weighed significantly less than vehicle-injected rats on day 14 . Vertical error bars represent the standard error of the difference between the means. Significant difference from vehicle: $* * * P<0.001$.

in the total number of myelinated axons $(P<0.05)$, the mean fascicle area $(P<0.05)$, and the mean endoneurial area (mean myelinated axon area subtracted from the mean fascicle area; $P<0.05$ ). Pyridoxine treatment also caused a significant reduction in the total neurofilament area $(P<0.001)$, the mean axon area (total neurofilament area divided by the total number of axons; $P<0.05$ ), and a trend toward a significant reduction in the mean myelinated axon area $(P=$ 0.068 ). No significant differences were present in total myelinated axon area, the total area of myelin, or the mean myelin area (difference between the mean myelinated axon area and the mean neurofilament area). The axon-to-myelin ratio, an indication of axonopathy or demyelination, was reduced in the pyridoxine-treated group, demonstrating toxicity associated with axonopathy rather than demyelination.

\section{Size distribution}

Size distribution, reflecting the number of myelinated nerve fibers of each size, is shown in Fig. 6. The size data were collated into area size bins from the smallest (1-10 $\left.\mu \mathrm{m}^{2}\right)$ up to the largest $\left(>200 \mu \mathrm{m}^{2}\right)$, and are presented as a percentage of the total number of fibers counted for each animal. For clarity, the data were further condensed into 25 $\mu \mathrm{m}^{2}$ area size bins: $1-25,25.1-50,50.1-75$, up to $>200$ $\mu \mathrm{m}^{2}$. The size distribution revealed a pyridoxine-mediated increase in the percentage of small-diameter (1-25 and $25.1-50 \mu \mathrm{m}^{2}$ ) fibers together with a relative decrease in the percentage of larger-diameter fibers $\left(50.1 \mu \mathrm{m}^{2}\right.$ and above), that is, an overall shift to the left. Nerve fibers from pyridoxine-treated rats showed a significant reduction in the percentage of fibers in the $50.1-75 \mu \mathrm{m}^{2}[F=5.291, d f$ (1,13), $P=0.04]$ and the $100.1-125 \mu^{2}[F=4.391, d f$ $(1,13), P=0.05]$ area size bins, and a trend toward a significant pyridoxine-mediated increase in the percentage of fibers in the $1-25 \mu \mathrm{m}^{2}$ size bin $[F=4.247, d f(1,13), P=$
0.062]. When this smallest area size bin was expanded (see Fig. 6, inset), the increase in the percentage of fibers in the smallest size bin $\left(1-10 \mu \mathrm{m}^{2}\right)$ reached significance $[F=6.113, d f(1,13), P=0.029]$.

\section{Stereological quantification of neurons in the dorsal root ganglion}

The mean total area of A and B cells in the DRG of vehicle- and pyridoxine-treated animals is shown in Fig. 7. The mean area of the ganglion was unchanged following pyridoxine treatment (vehicle $=1.02 \pm 0.19 \mathrm{~mm}^{2}(n=4)$ and pyridoxine $\left.=0.99 \pm 0.30 \mathrm{~mm}^{2}(n=7)\right)$. The pyridoxine-treated group showed a significant reduction in the mean area of A and B cells in the dorsal root ganglion $(F=16.589, d f(1,10), P=0.003$; Fig. 7$)$.

\section{Discussion}

This study provides a quantitative characterization of the morphological and electrophysiological consequences of pyridoxine intoxication. The results of this study provide further evidence that the primary site of injury in pyridoxine neurotoxicity is in the soma of neurons of the DRG with consequences in the axonal integrity of the long myelinated fibers in the sciatic nerve. This is strongly supported by the electrophysiological data showing that pyridoxine intoxication produced a severe attenuation (or loss) of the $\mathrm{H}$ wave amplitude with no observable change in $\mathrm{M}$ wave amplitude.
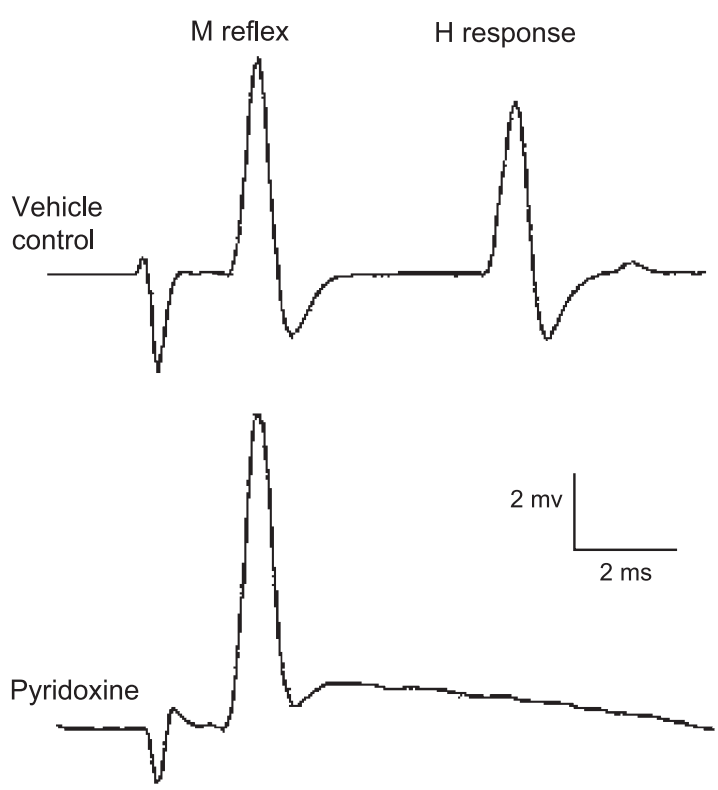

Fig. 4. Electrophysiological measures of sensory and motor function in pyridoxine-intoxicated and vehicle-injected rats. EMG potentials recorded after near sciatic nerve stimulation illustrating the $\mathrm{M}$ wave and $\mathrm{H}$ reflex are shown for a representative animal in the each group. Note the absence of the $\mathrm{H}$ reflex with pyridoxine administration. 

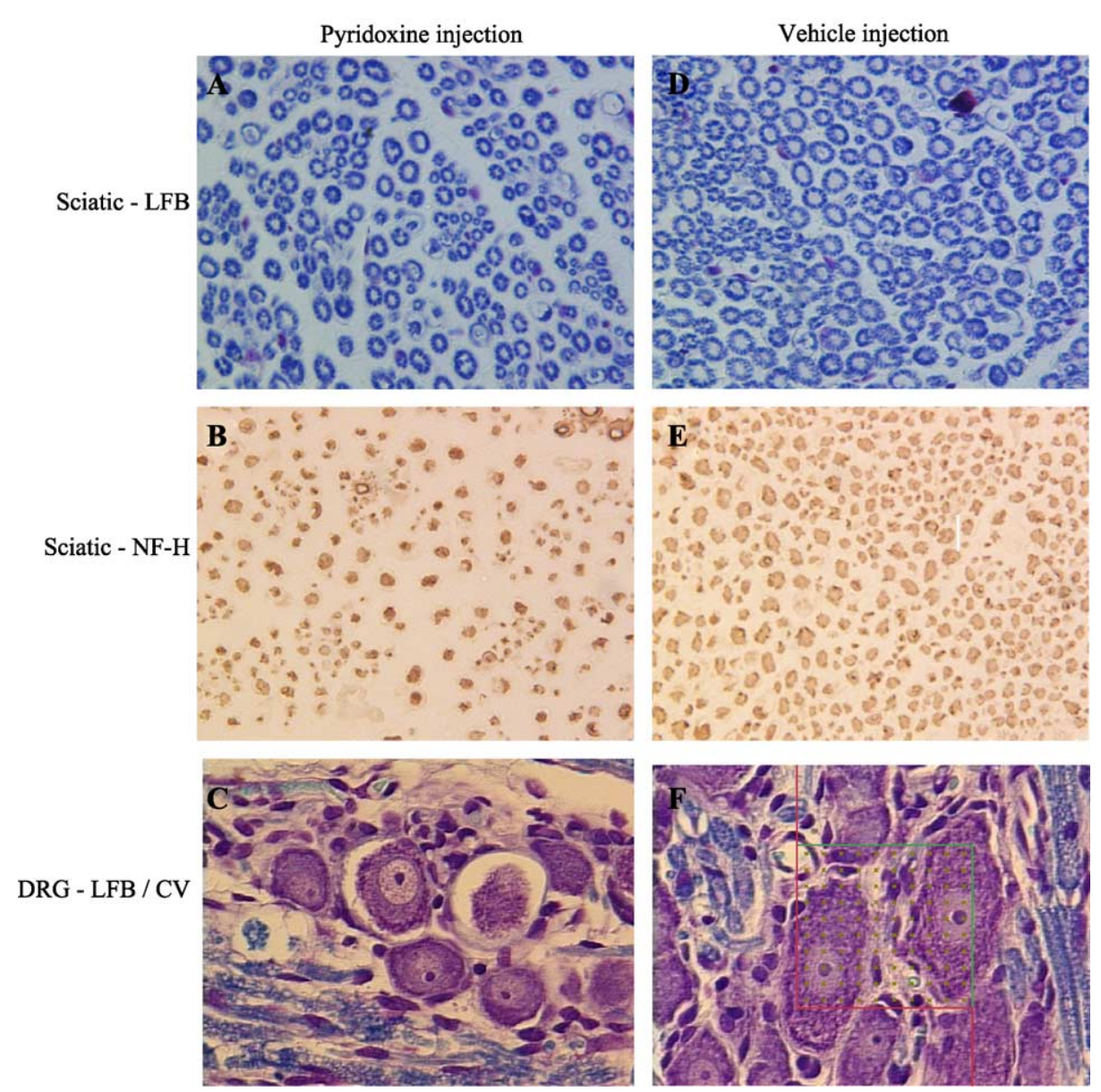

Fig. 5. Representative transverse sections of sciatic nerve and L4 or L5 dorsal root ganglia. Cross sections of sciatic nerve after 14 days of pyridoxine intoxication (A-B) or vehicle (D-E) injection showing luxol fast blue staining (LFB) for myelin (A and D) and neurofilament-positive immunoreactivity (NFH) for the axon (B and E). Degenerating fiber profiles are seen in the pyridoxine animals consisting of collapsed and irregularly shaped myelin sheaths, an increased number of smaller-diameter fibers, and endoneurium (connective tissue between individual nerve fibers). Cross sections of DRG from pyridoxine (C)- and vehicle (F)-treated animals stained with LFB and cresyl violet (CV). Degenerating profiles are seen in the intoxicated animals, specifically smaller cell bodies with evidence of intracytoplasmic vacuolation and increased numbers of satellite cells. The vehicle animal shows the normal DRG phenotype (F); large A-cells with well-defined Nissl granules and light nuclei, with one dark central nucleolus, and the smaller and darker B-cells that generally contain multiple nucleoli located peripherally.

Such decrements in $\mathrm{H}$ wave amplitude were associated with a reduction in sensory conduction velocity and a small fiber involvement.
Our observations at light microscope levels suggest that pyridoxine intoxication selectively targets the large-diameter A-cells of the DRG with consequential axonal atrophy of

Table 1

Stereological correlates examined in sciatic nerve sections after pyridoxine intoxication compared with vehicle treatment

\begin{tabular}{lccl}
\hline Variable & Vehicle-injected group & Pyridoxine-injected group & Statistics $d f(1,13)$ \\
\hline Total number of myelinated axons & $8174.65 \pm 4308.75$ & $14070.90 \pm 3897.04$ & $F=7.210 ; P=0.02^{*}$ \\
Mean myelinated axon area $\left(\mu \mathrm{m}^{2}\right)$ & $242.73 \pm 142.92$ & $131.17 \pm 34.59$ & $F=4.029 ; P=0.068$ \\
Mean fascicle area $\left(\mu \mathrm{m}^{2} \times 10^{6}\right)$ & $0.71 \pm 0.37$ & $1.10 \pm 0.17$ & $F=6.510 ; P=0.025^{*}$ \\
Total axon + myelin area $\left(\mu \mathrm{m}^{2} \times 10^{6}\right)$ & $1.68 \pm 0.84$ & $1.89 \pm 0.81$ & $F=0.236 ; P=\mathrm{NS}$ \\
Total neurofilament area $\left(\mu \mathrm{m}^{2} \times 10^{6}\right)$ & $0.25 \pm 0.05$ & $0.13 \pm 0.02$ & $F=31.330 ; P=0.0001^{* * *}$ \\
Total myelin area $\left(\mu \mathrm{m}^{2} \times 10^{6}\right)$ & $1.42 \pm 0.84$ & $1.75 \pm 0.80$ & $F=0.585 ; P=\mathrm{NS}$ \\
Mean endoneurial area $\left(\mu \mathrm{m}^{2} \times 10^{6}\right)$ & $0.71 \pm 0.37$ & $1.11 \pm 0.17$ & $F=6.511 ; P=0.025^{*}$ \\
Mean myelin area $\left(\mu \mathrm{m}^{2}\right)$ & $196.57 \pm 129.80$ & $121.28 \pm 3524$ & $F=2.193 ; P=\mathrm{NS}$ \\
Mean axon area $\left(\mu \mathrm{m}^{2}\right)$ & $46.16 \pm 41.92$ & $9.89 \pm 2.42$ & $F=5.221 ; P=0.041^{*}$ \\
Axon-to-myelin ratio & $0.29 \pm 0.26$ & $0.09 \pm 0.03$ & $F=4.148 ; P=0.064$ \\
\hline
\end{tabular}

Values are group means \pm standard deviation. Significant difference from vehicle group.

* $P<0.05$.

*** $P<0.001$. 


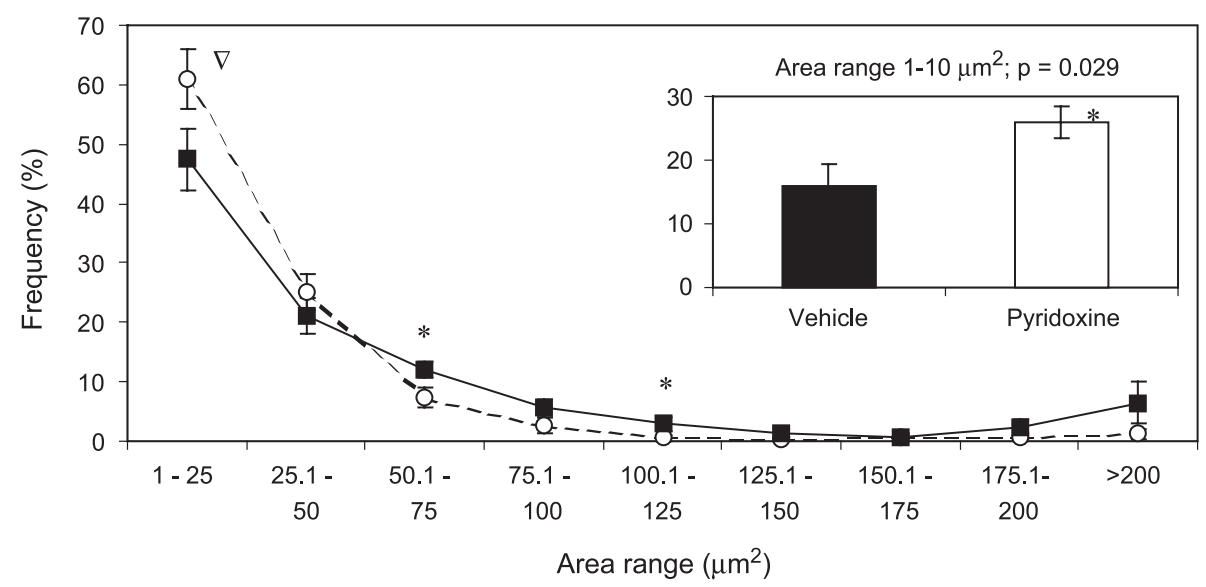

Fig. 6. Size frequency distribution showing the percentage of sciatic nerve fibers within specified area ranges following pyridoxine intoxication (-O-) or vehicle treatment (- - -) and, (inset) the percentage of fibers in the smallest area range; $1-10 \mu \mathrm{m}^{2}$. Vertical error bars represent the standard error of the difference between the means. Significant difference from vehicle: $* P<0.05$, and trend toward a significant difference from vehicle: $\nabla P=0.062$.

the larger-diameter myelinated fibers within the sciatic nerve. Specifically, pyridoxine toxicity appears to result in a significant sciatic nerve axonopathy, an increase in the total number of myelinated fibers caused by a shift of the size distribution from large to small diameter fibers, and an increased endoneurial area, which is likely related to the axonal degeneration together with the increased frequency of small diameter fibers. The axon-to-myelin ratio supports the view that pyridoxine toxicity is associated with axonopathy rather than demyelination. Initial observations suggested that there were fewer DRG cells with the classic A-cell morphology in pyridoxine-treated animals than in the vehicle-treated group. The criteria used for the morphological evaluation was dependent on the ability to identify the cell profile as a "classic DRG neuron". As the atrophy was severe, degenerating neurons had to possess the remains of Nissl staining (rough endoplasmic reticulum) with a clear cell membrane and be surrounded by satellite cells to be counted, even though the nucleus or nucleoli in many cases was not recognizable or not present in the particular cut plane of the section. There was a high degree of cytoplasmic vacuolization of the large neurons. The vacuoles appeared to fuse and form large cavities, which reduced the cytoplasm in

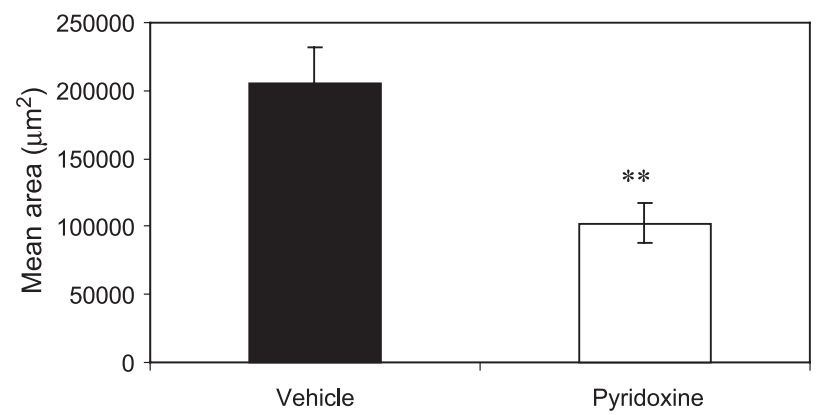

Fig. 7. Mean area $( \pm \mathrm{SD})$ of A- and B-cells in the dorsal root ganglion of vehicle- and pyridoxine-intoxicated animals. Vertical error bars represent the standard error of the difference between the means. Significant difference from vehicle: ${ }^{* *} P<0.01$. some cases to a thin rim. Pyridoxine-intoxicated animals also showed a dramatic increase in the number of satellite cells. Structures similar to ghost cells (Edwards and Tolkovsky, 1994), in which the only cytoplasmic features were the remains of condensed DNA, were evident in pyridoxine animals and were not counted. Stereological quantification confirmed a significant pyridoxine-mediated reduction in the mean area of A- and B-cells compared to the vehicle-treated group. A previous study used designbased stereology to quantify the total number and area of Aand B-cells in the DRG (Tandrup, 1993) in normal, untreated rats. Pyridoxine is a toxin known to target large fiber sensory neurons (Albin et al., 1987; Krinke and Fitzgerald, 1988; Schaeppi and Krinke, 1982; Windebank et al., 1985; Xu et al., 1989). Therefore, it was our expectation that pyridoxine would cause a selective reduction in A-cells in DRG. However, quantification of A- and B-cells individually would assume that one could distinguish between atrophied A-cells and B-cells, particularly difficult if the two-dimensional area estimation is of profiles where the nucleolus may not be visible. To avoid this confound, we quantified the mean area of A- and B-cells together to increase confidence in the interpretation that pyridoxine causes overall changes in both types of DRG cells.

The rationale for selecting pyridoxine to produce an animal model of large-fiber neuropathy was based on several factors, including the selective and severe neurotoxic actions of this compound on large DRG neurons in rodents (Xu et al., 1989), dogs (Schaeppi and Krinke, 1982), and humans (Albin et al., 1987). Pyridoxine has been shown to produce clear behavioral, electrophysiological, and anatomical deficits, without overt systemic morbidity as in cisplatin-induced neuropathy. Our interest in this particular animal model of sensory neuropathy was that the large-fiber neurodegeneration reflects one aspect of clinical diabetic peripheral neuropathy and would therefore be of use for evaluating the possible neurotrophic-neuroprotective properties of novel compounds currently in development for 
type 2 diabetes mellitus. These studies were developed according to the specifications required by current U.S. Environmental Protection Agency regulatory guidelines (U.S. EPA, 1998; for a recent review, see Garman et al., 2001). Although these guidelines apply strictly to developmental neurotoxicity studies, careful qualitative and quantitative morphologic study of both peripheral nerves and ganglia applies generally to the morphometric assessment of toxicity and therapeutic management of diseases involving the peripheral nervous system.

The neurotoxic mechanism of excess pyridoxine is not known, but several theories exist. The core structure of vitamin $\mathrm{B}_{6}$ is the pyrimidine ring, and the family of pyrimidines is known to have structurally dependent neurotoxic potential. High circulating levels of pyridoxine in the blood are thought to have a direct toxic effect on neurons. The restriction of susceptible cells to peripheral ganglia is likely associated with the lower ("leaky") bloodnerve barrier in such regions. In contrast, the presence of a more complete blood-brain barrier together with the presence of a saturable pyridoxine transport system may protect neurons in the brain and spinal cord from high circulating levels of pyridoxine. Interestingly, deficiency of this vitamin also elicits a neuropathy. Megadoses of pyridoxine may saturate hepatic pyridoxol kinase and pyridoxine phosphate oxidase, enzymes that convert the vitamin to the active form, pyridoxine phosphate. This allows the inactive form pyridoxine to act as a competitive inhibitor to pyridoxol phosphate at the neuronal level. Other possible mechanisms include involvement of excess pyridoxine in local chelation of magnesium or the formation of quinone methide, which attacks polar and unsaturated groups (Jortner, 2000).

In humans, peripheral neuropathies of various etiologies comprise a heterogeneous group of disorders vis-à-vis clinical manifestations and prognosis. The manifestation of clinical symptoms depends on the types of peripheral nerves involved, the dose and duration of exposure to the toxic agent, and the presence of any inherited factors. For example, diabetic neuropathy, peripheral nerve injury, or toxic neuropathies that arise as consequences of chemotherapy cause a spectrum of neuropathies. These pathologies are attributable to involvement of multiple classes of sensory or motor fibers. In contrast, the toxic neuropathies caused by pyridoxine produce a more homogeneous syndrome with selective damage to sensory fibers as shown here and in previous studies of experimental animals and humans dosed with pyridoxine. Regardless of the cause of injury, to date, there is no effective treatment for peripheral neuropathies.

Humans dosed with pyridoxine develop a sensory neuropathy similar to that produced in experimental animals (Albin et al., 1987; Parry and Bredesen, 1985; Schaumburg et al., 1983). Histopathological and electrophysiological data from both experimental animal and clinical studies involving megavitaminosis support the view that pyridoxine intoxication principally affects large primary sensory neurons, with secondary effects on their central and peripheral processes within the spinal nerves, the trigeminal nerve, and the spinal cord (Krinke et al., 1980, 1985; Parry and Bredesen, 1985; Schaumburg et al., 1983; Windebank et al., 1985; Xu et al., 1989). However, a previous study in humans demonstrated small fiber dysfunction in addition to the large fiber deficit (Berger et al., 1992). This study demonstrated that after low doses of pyridoxine human subjects developed small-fiber dysfunction as the earliest and predominant abnormality, with sensory modalities subserved by large-diameter, thickly myelinated fibers either unaffected or affected later than small fiber function. The authors reported that higher doses of pyridoxine led to degeneration of both large and small fibers. Targeting of small fibers by pyridoxine intoxication has also been reported in animal studies (Callizot et al., 2001). At a dose consistent with that used in the present study, Callizot et al. demonstrated a proprioceptive deficit caused by sensory neuropathy involving large fibers combined with involvement of small fibers evidenced by decreased sensory conduction velocity and altered thermosensitivity. Qualitative morphological analyses of fibers emerging from L4 and L5 indicated that pyridoxine intoxication spared sensory neuron cell bodies in DRG, in clear contrast with our data. Therefore, our findings of reduced sensory conduction velocity and spared motor function corroborate the involvement of a small fiber deficit, providing further support for the human study conducted by Berger et al. (1992). It is possible that the small fiber dysfunction may have been overlooked in previous clinical studies, either because the deficits were mild or because they were masked by concurrent, clinically more obvious large fiber dysfunction.

In the absence of quantitative data, Helgren et al. (1997) demonstrated that pyridoxine intoxication caused degeneration of primary afferent fibers in the dorsal column of the spinal cord combined with modest loss of L4-L5 sensory neurons. This finding accompanied a decrement in $\mathrm{H}$ wave amplitude, without loss of sensory conduction velocity, in clear contrast with our data and previous studies (Krinke et al., 1985; Windebank et al., 1985) that reported atrophy and death of DRG neurons and degeneration of their peripheral axonal processes in response to pyridoxine intoxication.

These experimental studies provide strong support for the view that pyridoxine toxicity results in the degeneration of peripheral sensory ganglia, particularly large neurons with long, heavily myelinated processes. At this time, the metabolic consequences of pyridoxine toxicity remain unclear. Pyridoxine toxicity likely results from dosedependent interference with neuronal metabolism. Highdose administration produces ataxia with necrosis of DRG neurons and lower doses produce cell body and axonal atrophy without discernable clinical sequelae. Between these ranges exists a spectrum of injury, with the earliest manifestations occurring at the level of the cell body. While 
megadose exposure results in metabolic failure in a selected population of DRG neurons with consequent neuronal and axonal necrosis, lower doses inflict a sublethal metabolic injury leading to cell body and axonal atrophy. The cell body manifests this damage as cytoplasmic alterations including vacuolization, increased dense bodies, neurofilament aggregates, and chromatolysis. More advanced neurotoxic changes lead to neuronal death with phagocytosis by satellite cells.

An early morphological correlate of pyridoxine neurotoxicity appears to be the accumulation of neurofilament with microtubule-neurofilament dissociation in the cell body and the proximal myelinated processes of the DRG in the absence of extensive vacuolization (Montpetit et al., 1988). Neurofilaments are major determinants of axon caliber in large myelinated nerve fibers (Hoffman et al., 1987; Kriz et al., 2000). The control of axonal caliber has a functional significance because diameter is the principle determinant of conduction velocity in myelinated nerve fibers (Hoffman et al., 1987; Waxman, 1980). The observed cytoskeletal disruption with pyridoxine intoxication may be related to an increased rate of neurofilament protein synthesis (Montpetit et al., 1988; Xu et al., 1989) rather than mechanical obstruction of transport phenomena (Krinke et al., 1985). The biochemical basis of the observed selective involvement of the peripheral sensory system is not known, although the toxicity of pyridoxine appears related to its concentration in the DRG, which lacks a blood-nerve barrier (Schaumburg et al., 1983). The molecular basis for the selectivity of pyridoxine toxicity for sensory neurons may be due to biochemically significant differences in pyridoxine intermediary metabolism rather than differences in rates of intracellular accumulation. Without doubt, multiple factors including dose and rate of administration, differential neuronal vulnerability, and species susceptibility are major determinants in the pathogenesis of pyridoxine neuropathy.

In summary, pyridoxine-induced sensory neuropathy provides a valuable addition to current models of experimental sensory neuropathies used in preclinical research for evaluating novel putative neuroprotective compounds as potential therapeutics for human peripheral neuropathies. With the recently developed design-based stereological techniques, it is now possible to eliminate many of the methodological problems and biases associated with previous studies. Thus for the first time, we have used stereology in conjunction with electrophysiology to quantify the neuropathy induced by pyridoxine intoxication in the soma of neurons in the DRG and the axons of the long myelinated fibers in the sciatic nerve. The disparate existing literature suggests that the dose of pyridoxine used is the major determinant in the specificity of the neuropathy produced, such that higher degrees of intoxication may incorporate small fiber degeneration in addition to large fiber degeneration and sensory neuron atrophy. The combined stereological and electrophysiological techniques in this study demonstrate a general approach for understanding the neurobiological factors underlying peripheral nerve degeneration as well as assessing the efficacy of therapeutic approaches for the management of peripheral neuropathy.

\section{Acknowledgments}

We would like to thank Dr. Donald K. Ingram, Laboratory of Experimental Gerontology for use of the Stereologer system. This work was supported by the National Institute on Aging Intramural Research Program.

\section{References}

Albin, R.L., Albers, J.W., Greenberg, H.S., Townsend, J.B., Lynn, R.B., Burke Jr., J.M., Alessi, A.G., 1987. Acute sensory neuropathyneuronopathy from pyridoxine overdose. Neurology 37, 1729-1732.

Berger, A.R., Schaumburg, H.H., Schroeder, C., Apfel, S., Reynolds, R., 1992. Dose response, coasting, and differential fiber vulnerability in human toxic neuropathy: a prospective study of pyridoxine neurotoxicity. Neurology 42, 1367-1370.

Bowe, C.M., Veale, K., 1988. Sensitivity to 4-aminopyridine observed in mammalian sciatic nerves during acute pyridoxine-induced sensory neuropathy and recovery. Exp. Neurol. 100, 448-458.

Callizot, N., Warter, J.M., Poindron, P., 2001. Pyridoxine-induced neuropathy in rats: a sensory neuropathy that responds to 4-methylcatechol. Neurobiol. Dis. 8, 626-635.

Dalton, K., Dalton, M.J., 1987. Characteristics of pyridoxine overdose neuropathy syndrome. Acta Neurol. Scand. 76, 8-11.

Edwards, S.N., Tolkovsky, A.M., 1994. Characterization of apoptosis in cultured rat sympathetic neurons after nerve growth factor withdrawal. J. Cell Biol. 124, 537-546.

Foca, F.J., 1985. Motor and sensory neuropathy secondary to excessive pyridoxine ingestion. Arch. Phys. Med. Rehabil. 66, 634-636.

Garman, R.H., Fix, A.S., Jortner, B.S., Jensen, K.F., Hardisty, J.F., Claudio, L., Ferenc, S., 2001. Methods to identify and characterize developmental neurotoxicity for human health risk assessment. II: neuropathology. Environ. Health Perspect. 109 (Suppl. 1), 93-100.

Hanrahan, J.P., Gordon, M.A., 1984. Mushroom poisoning. Case reports and a review of therapy. JAMA 251, 1057-1061.

Hoffman, P.N., Cleveland, D.W., Griffin, J.W., Landes, P.W., Cowan, N.J., Price, D.L., 1987. Neurofilament gene expression: a major determinant of axonal caliber. Proc. Natl. Acad. Sci. U. S. A. 84, 3472-3476.

Hoover, D.M., Carlton, W.W., Henrikson, C.K., 1981. Ultrastructural lesions of pyridoxine toxicity in beagle dogs. Vet. Pathol. 18, 769-777.

Jensen, G.B., Pakkenberg, B., 1993. Do alcoholics drink their neurons away? Lancet 342, 1201-1204.

Jensen Vedel, E.B., Gundersen, H.J.G., 1993. The rotator. J. Microsc. 170, $35-44$.

Jortner, B.S., 2000. Mechanisms of toxic injury in the peripheral nervous system: neuropathologic considerations. Toxicol. Pathol. 28, 54-69.

Krinke, G.J., Fitzgerald, R.E., 1988. The pattern of pyridoxine-induced lesion: difference between the high and the low toxic level. Toxicology 49, $171-178$.

Krinke, G., Schaumburg, H., Spencer, P., et al., 1980. Pyridoxine megavitaminosis produces degeneration of peripheral sensory neurons (sensory neuropathy) in the dog. Neurotoxicology 2, 13-24.

Krinke, G., Naylor, D.C., Skorpil, V., 1985. Pyridoxine megavitaminosis: an analysis of the early changes induced with massive doses of vitamin B6 in rat primary sensory neurons. J. Neuropathol. Exp. Neurol. 44, $117-129$ 
Kriz, J., Zhu, Q., Julien, J.P., Padjen, A.L., 2000. Electrophysiological properties of axons in mice lacking neurofilament subunit genes: disparity between conduction velocity and axon diameter in absence of NF-H. Brain Res. 885, 32-44.

Larsen, J.O., 1998. Stereology of nerve cross sections. J. Neurosci. Methods 85, 107-118.

Larsen, J.O., Thomsen, M., Haugland, M., Sinkjaer, T., 1998. Degeneration and regeneration in rabbit peripheral nerve with long-term nerve cuff electrode implant: a stereological study of myelinated and unmyelinated axons. Acta Neuropathol. (Berl.) 96, 365-378.

Montpetit, V.J., Clapin, D.F., Tryphonas, L., Dancea, S., 1988. Alteration of neuronal cytoskeletal organization in dorsal root ganglia associated with pyridoxine neurotoxicity. Acta Neuropathol. (Berl.) 76, 71-81.

Mouton, P.R., 2002. Principles and Practices of Unbiased Stereology: An Introduction for Bioscientists. The Johns Hopkins Univ. Press, Baltimore.

Nisar, M., Watkin, S.W., Bucknall, R.C., Agnew, R.A., 1990. Exacerbation of isoniazid induced peripheral neuropathy by pyridoxine. Thorax 45 , $419-420$.

Parry, G.J., Bredesen, D.E., 1985. Sensory neuropathy with low-dose pyridoxine. Neurology 35, 1466-1468.

Phillips, W.E., Mills, J.H., Charbonneau, S.M., Tryphonas, L., Hatina, G.V., Zawidzka, Z., Bryce, F.R., Munro, I.C., 1978. Subacute toxicity of pyridoxine hydrochloride in the beagle dog. Toxicol. Appl. Pharmacol. 44, 323-333.

Schaeppi, U., Krinke, G., 1982. Pyridoxine neuropathy: correlation of functional tests and neuropathology in beagle dogs treated with large doses of vitamin B6. Agents Actions 12, 575-582.
Schaumburg, H.H., Spencer, P.S., 1979. Toxic neuropathies. Neurology 29 , $429-431$.

Schaumburg, H., Kaplan, J., Windebank, A., Vick, N., Rasmus, S., Pleasure, D., Brown, M.J., 1983. Sensory neuropathy from pyridoxine abuse. A new megavitamin syndrome. N. Engl. J. Med. 309, 445-448.

Schionning, J.D., Larsen, J.O., 1997. A stereological study of dorsal root ganglion cells and nerve root fibers from rats treated with inorganic mercury. Acta Neuropathol. (Berl.) 94, 280-286.

Schionning, J.D., Larsen, J.O., Eide, R., 1998a. A stereological study of dorsal root ganglion cells and nerve root fibers from rats exposed to mercury vapor. Acta Neuropathol. (Berl.) 96, 185-190.

Schionning, J.D., Larsen, J.O., Tandrup, T., Braendgaard, H., 1998b. Selective degeneration of dorsal root ganglia and dorsal nerve roots in methyl mercury-intoxicated rats: a stereological study. Acta Neuropathol. (Berl.) 96, 191-201.

Tandrup, T., 1993. A method for unbiased and efficient estimation of number and mean volume of specified neuron subtypes in rat dorsal root ganglion. J. Comp. Neurol. 329, 269-276.

U.S. EPA, 1998. Developmental Neurotoxicity Study. U.S. Environmental Protection Agency, Washington, DC.

Waxman, S.G., 1980. Determinants of conduction velocity in myelinated nerve fibers. Muscle Nerve 3, 141-150.

Windebank, A.J., Low, P.A., Blexrud, M.D., Schmelzer, J.D., Schaumburg, H.H., 1985. Pyridoxine neuropathy in rats: specific degeneration of sensory axons. Neurology $35,1617-1622$.

Xu, Y., Sladky, J.T., Brown, M.J., 1989. Dose-dependent expression of neuronopathy after experimental pyridoxine intoxication. Neurology $39,1077-1083$. 